\title{
Interference between Competing Pathways in Atomic Harmonic Generation
}

\author{
A. C. Brown, ${ }^{1}$ S. Hutchinson, ${ }^{1}$ M. A. Lysaght, ${ }^{2}$ and H. W. van der Hart ${ }^{1}$ \\ ${ }^{1}$ Centre for Theoretical Atomic, Molecular and Optical Physics, Queen's University Belfast, Belfast, BT7 1NN, United Kingdom \\ ${ }^{2}$ Irish Centre for High End Computing, Tower Building, Grand Central Quay, Dublin 2, Ireland
}

(Received 12 October 2011; published 9 February 2012)

\begin{abstract}
We investigate the influence of the autoionizing $3 s 3 p^{6} n \ell$ resonances on the fifth harmonic generated by 200-240 nm laser fields interacting with Ar. To determine the influence of a multielectron response we develop the capability within time-dependent $R$-matrix theory to determine the harmonic spectra generated. The fifth harmonic is affected by interference between the response of a $3 s$ electron and the response of a $3 p$ electron, as demonstrated by the asymmetric profiles in the harmonic yields as functions of wavelength.
\end{abstract}

DOI: 10.1103/PhysRevLett.108.063006

PACS numbers: 32.80.Rm, 31.15.A-, 42.65.Ky

Harmonic generation (HG) is one of the fundamental processes that can occur in light-matter interaction. The process is of strong technological interest, since it lies at the heart of ultrashort light pulse generation [1]. However, $\mathrm{HG}$ can also be used as a measurement tool, for example, to study molecular changes on the subfemtosecond time scale [2]. Recent articles have postulated various other applications of high harmonics, including electron interferometry and the tomographic imaging of electron orbitals [3]. HG has also been used experimentally to demonstrate the importance of electron-correlation in ultrafast molecular dynamics [4] and multielectron dynamics in atomic systems [5].

In order to probe the influence of multielectron dynamics on HG theoretically, we find it more expedient to consider atomic systems. Although detailed calculations for molecules are possible using sophisticated codes, it is much more difficult to identify the multielectron dynamics within a molecular system than within an atomic system. Even so, many methods developed for the determination of harmonic spectra in atoms rely on the single-activeelectron approximation [6,7]. Several methods have been developed for the description of two-electron systems $[8,9]$, but these methods are not easy to extend to general atoms. New methods are thus needed to gain insight into the influence of multielectron dynamics on harmonic spectra in general multielectron atoms.

At Queen's University Belfast we have developed timedependent $R$-matrix (TDRM) theory over the last five years expressly to study the behavior of multielectron atoms in short, intense light fields [10]. The advantage of this method in studying multielectron dynamics was shown in a pump-probe study of $\mathrm{C}^{+}$[11]: by varying the delay between the pump and probe pulses, oscillations in the total ionization yield were observed, which could be linked to the correlated motion of two electrons. Because of the interest in using harmonic spectra to observe multielectron dynamics, we have developed capability within the time-dependent $R$-matrix approach to determine harmonic spectra for a single atom.
As a first demonstration of this capability, we report on the influence of the $3 s 3 p^{6} n \ell$ Rydberg series on single atom harmonic spectra in Ar. In particular, we focus on the wavelength range for which the fifth harmonic coincides with these resonances: 200-240 $\mathrm{nm}$. Recent studies have focused on the parallels between photoionization and HG $[12,13]$, where, in particular, the Cooper minimum in $\mathrm{Ar}$ was investigated. However, minima in the photoionization cross section can also occur near window resonances, where resonances actually lead to a decrease in the photoionization cross section. The $3 s 3 p^{6} n \ell$ resonances are examples of window resonances [14]. Their decay towards $3 s^{2} 3 p^{5} \varepsilon \ell^{\prime}$ continua requires a good description of dielectronic repulsion. The harmonic yield will be determined by two interfering pathways (Fig. 1): excitation of a $3 s$ electron to $n p$, and excitation of a $3 p$ electron into the continuum. A multielectron calculation is essential to describe the harmonic yield accurately. This study thus provides a good test case.

To describe the extension to compute a system's harmonic response, we first give a brief overview of TDRM theory. A more thorough discussion can be found in [10].

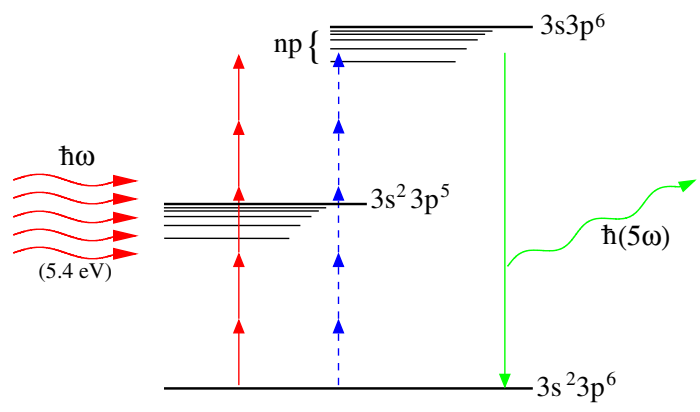

FIG. 1 (color online). The harmonic yield in Argon is determined by two competing pathways: absorption of at least three photons can excite a $3 p$ electron (red solid line) into the continuum, while absorption of at least five photons can excite a $3 s$ (blue dashed line) electron to the $n p$ Rydberg series converging onto the $3 s 3 p^{6}$ threshold. 
The time-dependent Schrödinger equation for an atom containing $(N+1)$ electrons, and nuclear charge $Z$, is

$$
i \frac{\partial}{\partial t} \Psi\left(\mathbf{X}_{N+1}, t\right)=H(t) \Psi\left(\mathbf{X}_{N+1}, t\right) .
$$

The Hamiltonian, $H$, contains both the nonrelativistic Hamiltonian of the $N+1$-electron atom or ion in the absence of the laser field and the laser interaction term. The laser field is described using the dipole approximation in the length form, and is assumed to be linearly polarized and spatially homogeneous. This form provides the most reliable ionization yields when only a limited amount of atomic structure is included [15].

To propagate the wave function in time, time is discretized into steps of $\Delta t$ such that

$$
t_{q}=q \Delta t, \quad q=0,1,2, \ldots
$$

Then, using the unitary Cayley form of the time evolution operator, $\exp [-i t H(t)]$, the wave function $\Psi$ in Eq. (1) at $t_{q+1}$ can be written in terms of the wave function at $t_{q}$

$$
\left[H\left(t_{q+1 / 2}\right)-E\right] \Psi\left(\mathbf{X}_{N+1}, t_{q+1}\right)=\boldsymbol{\Theta}\left(\mathbf{X}_{N+1}, t_{q}\right),
$$

where

$$
\boldsymbol{\Theta}\left(\mathbf{X}_{N+1}, t_{q}\right)=-\left[H\left(t_{q+1 / 2}\right)+E\right] \Psi\left(\mathbf{X}_{N+1}, t_{q}\right),
$$

and $H\left(t_{q+1 / 2}\right)$ is the time-dependent Hamiltonian at the midpoint. $E$ is given by $2 i / \Delta t$. In Eqs. (3) and (4) $\mathbf{X}_{N+1}=$ $\mathbf{r}_{1} \sigma_{1}, \mathbf{r}_{2} \sigma_{2}, \cdots, \mathbf{r}_{N+1} \sigma_{N+1}$ where $\mathbf{r}_{i} \sigma_{i}$ are the space and spin coordinates of the $i$ th electron. If the wave function at time $t=t_{q}$ is known, then Eq. (3) allows us to propagate the solution forward in time.

In $R$-matrix theory, configuration space is partitioned into two regions: an inner and an outer region. In the inner region, all electrons are within a distance $a_{\text {in }}$ of the nucleus, and all interactions between all electrons are taken into account. In the outer region, one electron has moved beyond a distance $a_{\text {in }}$, and exchange interactions between this electron and the electrons remaining close to the nucleus can be neglected. The outer electron thus experiences only the long-range multipole potential of the residual $N$-electron core and the laser field.

In the outer region, $a_{\mathrm{in}} \leq r_{N+1} \leq a_{\text {out }}$, we have

$$
\Psi\left(\mathbf{X}_{N+1}, t_{q+1}\right)=\sum_{p=1}^{n} \bar{\Phi}_{p}^{\gamma}\left(\mathbf{X}_{N} ; \hat{\mathbf{r}}_{N+1} \sigma_{N+1}\right) r_{N+1}^{-1} F_{p}\left(r_{N+1}\right),
$$

where $\bar{\Phi}_{p}^{\gamma}$ are channel functions which couple possible final states of the $\mathrm{Ar}^{+}$ion with spin and angular momentum of the ejected electron. The $F_{p}$ are the radial wave functions which describe the motion of the ejected electron in the $p$ th channel, where $p$ is a channel index.

We can now follow $R$-matrix procedures [16] to evaluate Eq. (3) at the boundary $a_{\text {in }}$ as a matrix equation

$$
\mathbf{F}\left(a_{\mathrm{in}}\right)=\mathbf{R}\left(a_{\mathrm{in}}\right) \overline{\mathbf{F}}\left(a_{\mathrm{in}}\right)+\mathbf{T}\left(a_{\mathrm{in}}\right),
$$

in which the wave function at the boundary is described in terms of its derivative, $\overline{\mathbf{F}}$, plus an inhomogeneous vector, $\mathbf{T}$, arising from the $\Theta$ term in Eq. (3). Equation (6) connects the inner and outer region wave function at the boundary $a_{\text {in }}$.

Given an initial inner region wave function, $\mathbf{R}$ and $\mathbf{T}$ are evaluated at the boundary. Subsequently, they are propagated outwards in space up to a boundary, $a_{\text {out }}$, where it can be assumed that the wave function $\mathbf{F}$ has vanished. The wave function vector $F$ is set to zero and then propagated inwards to the inner region boundary. Once the $F$ vector has been determined at each boundary point, the full wave function can be extracted from the $R$-matrix equations. Equation (3) then allows us to step this solution forward in time.

HG arises from the oscillating dipole moment induced by the laser field. This is given by the expectation value of the electric dipole operator,

$$
\mathbf{d}(t)=\langle\Psi(t)|-e \mathbf{Z}| \Psi(t)\rangle,
$$

where $e$ is the electronic charge and $\mathbf{Z}$ the total position operator along the laser polarization axis. The power spectrum of the emitted radiation is then given by the Fourier transform of either the dipole moment, its velocity or acceleration [17]. Many techniques involving high-order harmonics use the acceleration representation as it gives better resolution for the high-order peaks [18]. However, this has predominantly been verified for single-activeelectron systems, whereas in the multielectron TDRM method the length form of the dipole operator has proven to be far more reliable [15].

To describe Ar, we use the $R$-matrix basis developed for single photon ionization [19] with an inner region radius of 20 a.u. The set of continuum orbitals contains $60 \mathrm{~B}$ splines for each angular momentum, $\ell$, of the continuum electron. We use both the $3 s^{2} 3 p^{52} P^{o}$ and $3 s 3 p^{61} P^{o}$ states of $\mathrm{Ar}^{+}$as target states. The description of Ar includes all $3 s^{2} 3 p^{5} \epsilon \ell$ and $3 s 3 p^{6} \epsilon \ell$ channels up to $L_{\max }=15$. In the outer region we propagate $\mathbf{R}$ and $\mathbf{T}$ out to a radial distance of 1600 a.u. to prevent any reflections of the wave function from the outer region boundary. The outer region is divided into sectors of width 2 a.u. and each sector contains $35 B$ splines of order $k=9$ per channel. We use similar pulse shapes to those used in [10]: a 3 cycle $\sin ^{2}$ turn on or off for the electric field. Peak intensity is set at $2 \times 10^{13} \mathrm{~W} \mathrm{~cm}^{-2}$ with varying numbers of cycles at peak intensity; the time step is 0.1 a.u.

Figure 2 shows a typical power spectrum obtained for an incident pulse with a photon energy of $5.4 \mathrm{eV}$ and 25 cycles at peak intensity. As well as the three harmonic peaks, several other features are visible. The sideband structure of the harmonic peaks is due to the turn on or off of the laser pulse. In the photon energy region between $12.1-15.2 \mathrm{eV}$, 


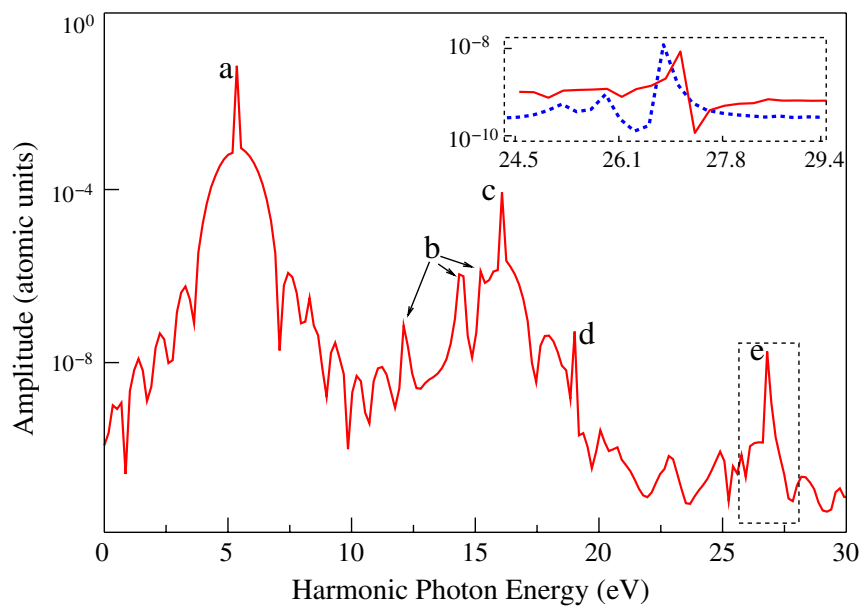

FIG. 2 (color online). The power spectrum produced by a 25 cycle, $2 \times 10^{13} \mathrm{~W} \mathrm{~cm}^{-2}$ laser pulse of photon energy $5.4 \mathrm{eV}$. The features labeled are (a) first harmonic peak, (b) effects due to $3 s^{2} 3 p^{5} n \ell$ Rydberg series, (c) third harmonic peak, (d) peak due to $3 s^{2} 3 p^{5} 4 p$ states plus a photon, and (e) fifth harmonic peak. Inset: The fifth-harmonic region of the power spectra for two 25 cycle pulses of photon energy $5.36 \mathrm{eV}$ (blue, dashed line), and $5.42 \mathrm{eV}$ (red, solid line). The change in the profile of the peaks is typical of the presence of a resonance in between these two photon energies.

several peaks are observed arising from the $3 s^{2} 3 p^{5} n \ell$ Rydberg series: $4 s$ at $12.1 \mathrm{eV}$, the $5 s / 3 d$ manifold at $14.5 \mathrm{eV}$, and the $4 d / 6 s$ at $15.1 \mathrm{eV}$. Higher manifolds are not separated at this pulse length. The third harmonic peak is clearly visible; it lies just above the $3 s^{2} 3 p^{5}$ threshold of $\mathrm{Ar}^{+}$. Its proximity to the threshold may mean that it still overlaps the Rydberg series leading up to this threshold. The peak at $18.8 \mathrm{eV}$ cannot be associated directly with any atomic structure but this energy corresponds to the energy of $3 s^{2} 3 p^{5} 4 p$ plus one $5.4 \mathrm{eV}$ photon. Finally, the fifth harmonic is visible at an energy of $26.9 \mathrm{eV}$.

The power spectrum was compared with calculations carried out using the $R$-matrix Floquet (RMF) method [20]. Good agreement was found at $248 \mathrm{~nm}$ for the first harmonic rate at intensities between 6.5 and $8.5 \times$ $10^{12} \mathrm{~W} \mathrm{~cm}^{-2}$. The RMF calculations also obtained thirdand fifth-harmonic rates within the $3 d / 5 s$ manifold. In RMF theory these states are separate, but this requires unfeasibly long pulses in TDRM theory. Thus no comparison is possible beyond the first harmonic.

The main interest in the present Letter is the fifth harmonic, which is strongly influenced by the $3 s 3 p^{6} n \ell$ Rydberg series. Photoionization experiments and calculations have demonstrated that the Rydberg series appears as window resonances [14,19]. Figure 2 also shows part of the power spectra at two distinct photo energies, $5.36 \mathrm{eV}$ and $5.42 \mathrm{eV}$. The resonance profile in the harmonic spectrum changes significantly. This is typical when a resonance lies in between these two photon energies. In order to assess these changes, the fifth-harmonic rate is determined over a

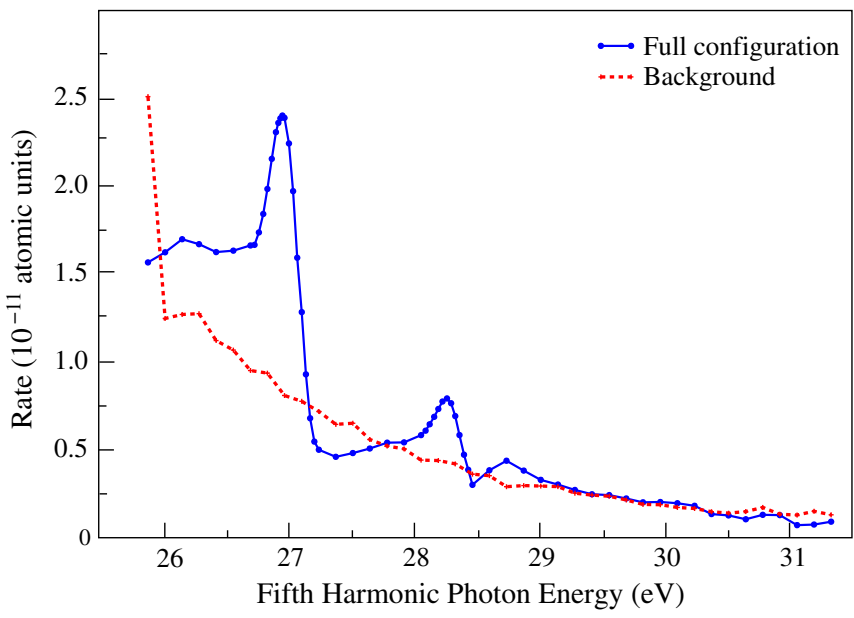

FIG. 3 (color online). The fifth-harmonic rate for $\mathrm{Ar}$ in a 25 cycle pulse of varying frequency, for a target with the full configuration (blue, solid line) and one without any contribution from the $3 s 3 p^{6} n / \varepsilon \ell$ series (red, dashed line). The resonances in the fifth-harmonic rate correspond to the $3 s 3 p^{6} 4 p(26.9 \mathrm{eV})$ and $3 s 3 p^{6} 5 p(28.3 \mathrm{eV})$ resonances.

range of photon energies. For each photon energy, we integrate the harmonic rate across the bandwidth of the harmonic peak. This approach yields the harmonic rates shown in Fig. 3. We can see resonances occurring at energies corresponding to the $3 s 3 p^{6} 4 p$ and $3 s 3 p^{6} 5 p$ states. Additional resonances may be present at higher photon energies, but the energy uncertainty makes their assessment difficult.

In order to obtain resonance parameters, calculations are also performed with a reduced basis: a $3 s^{2} 3 p^{5}$ singletarget-state description of $\mathrm{Ar}^{+}$, and an $\mathrm{Ar}$ basis which contains no $3 s 3 p^{6} n / \varepsilon \ell$ functions. This basis produces a smooth spectrum with a rate which can act as a background. Subtraction of this background rate then reveals a resonance profile due to the $3 s 3 p^{6} n \ell$ Rydberg series, from which resonance parameters can be established. The resonance profile in Fig. 4 was obtained for 25 cycle pulses at an intensity of $2 \times 10^{13} \mathrm{~W} \mathrm{~cm}^{-2}$, giving, within an accuracy of $10 \%$, an asymmetry parameter $q=-1.84$ and line width, $\Gamma=0.27 \mathrm{eV}$.

The resonance parameters differ significantly from those obtained in previous photoionization calculations [19]. The present resonance width is about a factor 4 larger than obtained previously, $0.068 \mathrm{eV}$. This is not too surprising, since the present calculations are carried out only over a finite laser pulse, which gives rise to a broadening of the resonance. The effects of this broadening can be estimated by carrying out calculations for different pulse lengths. Table I indeed shows that the resonance width decreases with increasing laser pulse length. In addition, the resonance may also broaden due to the intensity of the laser pulse. At higher intensities states can photoionize as well as autoionize. This leads to a reduction in the lifetime of the state, and hence a broadening. This effect is shown in 


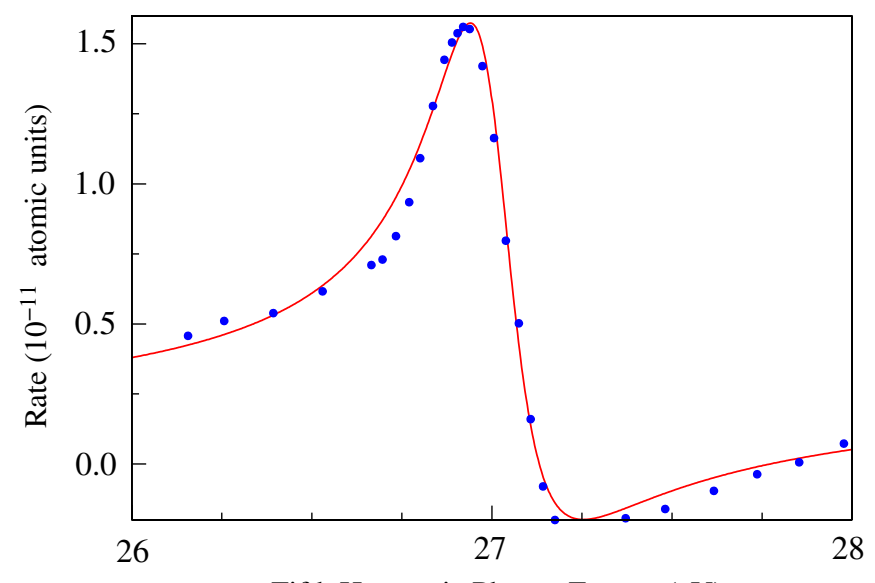

Fifth Harmonic Photon Energy (eV)

FIG. 4 (color online). The fifth-harmonic resonance profile for $\mathrm{Ar}$ in a 25 cycle laser pulse of intensity $2 \times 10^{13} \mathrm{~W} \mathrm{~cm}^{-2}$. The data (blue points) are fitted by a Fano profile (red line) with asymmetry parameter, $q=-1.84$ and line width $\Gamma=0.27 \mathrm{eV}$, and a linear background term.

Table II, where resonance parameters are compared as a function of peak intensity.

Figure 4 shows that at the fifth-harmonic stage, the resonance has a strong effect on the harmonic yields. The strong interference between the continuum and the resonance leads to an asymmetric profile with harmonic yields increased below and decreased above the resonance energy. Both the continuum and the resonant pathway to HG must therefore be accounted for. As these pathways involve the response of different electrons, it shows that a multielectron treatment of $\mathrm{HG}$ is necessary for $\mathrm{Ar}$ in the 200-240 nm wavelength range.

Table I shows that the asymmetry parameter is not affected significantly by the pulse duration. On the other hand, Table II shows that this parameter changes noticeably with the intensity of the laser pulse. This knowledge is important for modeling the harmonic response of a macroscopic system and the appearance of resonances over a particular energy distribution. However, it is difficult to analyze the parameter changes in detail. At an intensity of $5 \times 10^{13} \mathrm{~W} \mathrm{~cm}^{-2}$ the ponderomotive shift of the ground state means that at the fifth-harmonic resonance, the third harmonic lies very close to the $3 s^{2} 3 p^{5}$ threshold, and this may affect the profile.

TABLE I. The asymmetry parameters, line widths, and positions for the resonance profiles due to $2 \times 10^{13} \mathrm{~W} \mathrm{~cm}^{-2}$ laser pulses of various lengths accurate to within $\sim 10 \%$.

\begin{tabular}{lccc}
\hline \hline Peak cycles & $q$ parameter & Line width $(\mathrm{eV})$ & Position $(\mathrm{eV})$ \\
\hline 10 & -1.83 & 0.50 & 26.99 \\
15 & -1.82 & 0.40 & 27.01 \\
20 & -1.83 & 0.30 & 27.00 \\
25 & -1.84 & 0.27 & 27.01 \\
\hline \hline
\end{tabular}

TABLE II. The asymmetry parameters, line widths, and positions for the resonance profiles due to 25 cycle laser pulses of various intensities, accurate to within $\sim 10 \%$.

\begin{tabular}{lccc}
\hline \hline $\begin{array}{l}\text { Intensity } \\
\left(10^{13} \mathrm{~W} \mathrm{~cm}^{-2}\right)\end{array}$ & $q$ parameter & $\begin{array}{c}\text { Line width } \\
(\mathrm{eV})\end{array}$ & $\begin{array}{c}\text { Position } \\
(\mathrm{eV})\end{array}$ \\
\hline 2.0 & -1.84 & 0.27 & 27.01 \\
3.0 & -1.79 & 0.30 & 27.08 \\
5.0 & -1.31 & 0.32 & 27.27 \\
\hline \hline
\end{tabular}

In conclusion we have applied TDRM theory to study the appearance of the $3 s 3 p^{6} n \ell$ autoionizing resonances in the fifth harmonic for Ar. These window resonances show significant asymmetry within the fifth-harmonic spectrum, indicating interference between pathways involving the response of different electrons. This study provides a first demonstration of the new capability within time-dependent $R$-matrix theory to obtain harmonic spectra in multielectron atoms with electron-electron correlation fully included. This extension may enable further evaluation of whether and how evidence of multielectron dynamics can be found within harmonic spectra of atoms.

In the future, we hope to extend the approach to longer wavelengths and higher intensities. This will, however, require an assessment of the different gauges that can be used to obtain the harmonic spectrum, and extensive code development. The TDRM approach has proven its merit for strong-field ionization at wavelengths of $390 \mathrm{~nm}$ at $4 \times$ $10^{14} \mathrm{~W} \mathrm{~cm}^{-2}$ [16]. It will be of interest to export the determination of harmonic yields to the newly developed $R$ matrix with time-dependence codes [21], which may be better suited for longer wavelengths.

A.C. B. and S.H. acknowledge support from DEL NI under the programme for government. H.W.H. is supported by G/055816/1 from the ESPRC. M. A. L. acknowledges funding from the Numerical Algorithms Group (NAG) Ltd.

[1] P. M. Paul et al., Science 292, 1689 (2001).

[2] S. Baker et al., Science 312, 424 (2006).

[3] P. B. Corkum, Phys. Today 64, No. 3, 36 (2011).

[4] O. Smirnova et al., Nature (London) 460, 972 (2009).

[5] A. D. Shiner et al., Nature Phys. 7, 464 (2011).

[6] K. C. Kulander et al., Atoms in Intense Radiation Fields, edited by M. Gavrila (Academic Press, New York, 1992), pp. 247-300.

[7] K. J. Schafer and K. C. Kulander, Phys. Rev. Lett. 78, 638 (1997).

[8] J. S. Parker et al., Phys. Rev. Lett. 96, 133001 (2006).

[9] P. Lambropoulos, P. Maragakis, and J. Zhang, Phys. Rep. 305, 203 (1998).

[10] M. A. Lysaght, H. W. van der Hart, and P. G. Burke, Phys. Rev. A 79, 053411 (2009). 
[11] M. A. Lysaght, P. G. Burke, and H. W. van der Hart, Phys. Rev. Lett. 102, 193001 (2009).

[12] H. J. Wörner et al., Phys. Rev. Lett. 102, 103901 (2009).

[13] J. Higuet et al., Phys. Rev. A 83, 053401 (2011).

[14] R. P. Madden, D. L. Ederer, and K. Codling, Phys. Rev. 177, 136 (1969).

[15] S. Hutchinson, M. A. Lysaght, and H. W. van der Hart, J. Phys. B 43, 095603 (2010).
[16] H. W. van der Hart, M. A. Lysaght, and P. G. Burke, Phys. Rev. A 76, 043405 (2007).

[17] J. C. Baggesen and L. B. Madsen, J. Phys. B 44, 115601 (2011).

[18] T. F. Jiang and S. I. Chu Phys. Rev. A 46, 7322 (1992).

[19] P. G. Burke and K. T. Taylor, J. Phys. B 8, 2620 (1975).

[20] M. Plummer and C. J. Noble, J. Phys. B 35, L51 (2002).

[21] L. R. Moore et al., J. Mod. Opt. 58, 1132 (2011). 